\title{
A Humble Tribute to 50 Years of Fuzzy Sets
}

\author{
Luis Martínez ${ }^{\mathbf{1}}$, Jie Lu ${ }^{2}$ \\ ${ }^{1}$ Computer Sciences Department, University of Jaén, \\ Jaén, 23071, Spain \\ E-mail:martin@ujaen.es \\ ${ }^{2}$ Faculty of Engineering and Information Technology, University of Technology Sydney \\ PO Box 123, Broadway, NSW 2007, Australia \\ E-mail: jie.lu@uts.edu.au
}

\section{Preface}

2015 was the 50th anniversary of the first contribution on Fuzzy Sets ${ }^{1}$ and from the International Journal of Computational Intelligence Systems we want to take this opportunity to pay tribute to Lofti Zadeh, the father of Fuzzy Sets theory, which has guided many researchers and resulted in important advances in various fields of modern society. We also recognise and thank all the researchers who have devoted their careers to studying, developing and applying Fuzzy Sets theory across these 50 years. We want to show our respect, admiration and appreciation to all of them.

This Special Issue does not aim to simply be a comprehensive review of the history of Fuzzy Sets; rather, it intends to provide multiple views of these 50 years by incorporating contributions that either survey the use of Fuzzy Sets in different topics, or validate its application in real-world problems or its links with other theories. To achieve this, relevant and well-known researchers from the Fuzzy Sets and computational intelligence fields have contributed seven papers to this issue.

The first paper, Where Fuzzy Set Theory Reached in its 50th Year: A Comprehensive Literature Review, co-authored by Cengiz Kahraman, Sezi Ce- vik Onar and Basar Oztaysi, shows the evolution of Fuzzy Sets theory across this 50 -year history by first exploring the chronological evolution of publications related to fuzzy sets in diverse research areas and then recording the historical progress of ordinary fuzzy sets and their extensions. Lastly, the authors present a range of views on the future of fuzzy sets.

The paper Fuzzy Bi-level Decision-Making Techniques: A Survey, co-authored by Guangquan Zhang, Jialin Han and Jie Lu, provides a clear and systematic review of fuzzy bi-level decisionmaking techniques including models, approaches, algorithms and systems. It clusters related developments in technique into key categories, to support researchers and practitioners in their understanding of developments in theoretical research and applications in relation to fuzzy bi-level decision-making techniques.

Witold Pedrycz presents a general view of the area of fuzzy modeling in the paper entitled From Fuzzy Models to Granular Fuzzy Models. He elaborates on a new direction in system modeling by introducing the concept of granular models, which constitute a generalization of existing fuzzy models and, in contrast to existing models, generate results 
in the form of information granules. The development of granular models is completed through an optimal allocation of information granularity, which optimizes coverage criteria and the specificity of granular information. The appearance of granular models of type- 2 and type- $n$ is discussed along with an explanation of their formation.

The paper Classifying Image Analysis Techniques from their Output, co-authored by Carely Guada, Daniel Gomez, J. Tinguaro Rodríguez, Javier Yáñez and Javier Montero, discusses the most important image processing techniques and proposes classification based on the output provided by these methods. The authors also demonstrate the importance of fuzzy theory and fuzzy models to these techniques in addressing specific problems and obtaining good results.

In the paper entitled A View on Fuzzy Systems for Big Data: Progress and Opportunities, co-authored by Alberto Fernández, Cristobal J. Carmona, María J. del Jesus, and Francisco Herrera, examines the trends in big data, highlighting the tools and techniques that facilitate the migration of standard data mining systems to a new functional paradigm for working with Big Data problems. It points out the advantages of the techniques and approaches based on fuzzy systems and used in data mining for big data contexts. The authors express their views on expectations for the future in this context in relation to the design of those methods and approaches based on fuzzy sets.
Rosa M. Rodríguez, Alvaro Labella, and Luis Martínez present the paper entitled An Overview on Fuzzy Modelling of Complex Linguistic Preferences in Decision Making, a succinct review of the use of fuzzy modeling to elicit complex linguistic preferences in decision making problems that facilitate Computing with Words processes. They point out the strengths and weaknesses of the various proposals for modeling this type of information, as well as the open challenges that fuzzy-based approaches must face to improve the elicitation of the linguistic preferences of human beings.

Lastly, Stefania Marrara and Gabriella Pasi, in the paper Fuzzy Approaches to Flexible Querying in XML Retrieval, introduce fuzzy approaches for flexible querying in XML that aim to improve the expressiveness of query languages for XML data. They mainly focus on FleXy, a flexible extension of XQuery-FT and introduce PatentLight, a tool for patent retrieval that was developed to show the expressiveness power of FleXy.

We believe that this issue will provide a useful resource of ideas, techniques, and methodologies for additional research on fuzzy set theories and applications. We are grateful to the referees, whose valuable and much-appreciated work has contributed to the high quality of the papers published in this issue.

\section{References}

1. L.A. Zadeh. Fuzzy sets. Information and Control, 8:338-353, 1965. 